\title{
ORIGINAL
}

\section{INFECCIÓN NOSOCOMIAL EN PACIENTES QUIRÚRGICOS. PROBLEMAS DE MEDICIÓN Y DE COMPARACIÓN DE RESULTADOS *}

\author{
Carlos Aibar Remón, María José Rabanaque Hernández y Luis Ignacio Gómez López.
}

Departamento de Microbiología. Medicina Preventiva y Salud Pública. Facultad de Medicina. Universidad de Zaragoza.

* Este trabajo fue financiado con una beca del Consejo Asesor de Investigación de la Diputación General de Aragón.

\section{RESUMEN}

Fundamento: Al estudiar la frecuencia de infección nosocomial (IN), llama la atención la variabilidad de los datos de los estudios. En las estimaciones se utilizan diferentes indicadores de frecuencia y criterios de infección que dificultan la comparabilidad de los trabajos. El objetivo de esta investigación fue estimar la frecuencia de infección hospitalaria, utilizando diferentes indicadores para comparar los resultados.

Métodos: Se realizó un estudio prospectivo incluyendo los pacientes ingresados en cuatro servicios quirúrgicos durante un año. Se utilizaron los siguientes indicadores: proporción de pacientes infectados, incidencia acumulada de infección y densidad de incidencia. Las infecciones se detcctaron mediante búsqueda activa y se incluyeron las adquiridas en las UCIs y las diagnosticadas tras el alta hospitalaria.

Resultados: El 14,5\% de los pacientes padecieron IN y el $5 \%$ de las infecciones fueron diagnosticadas tras el alta. En el $38,5 \%$ de las infecciones no se solicitó estudio microbiológico. El servicio de cirugía general fue el que presentó las cifras más elevadas en los tres indicadores, no obstante las diferencias entre servicios se modificaron en función del indicador utilizado.

Conclusiones: El porcentaje real de pacientes con IN es superior a los valores aportados por los sistenias habituales de vigilancia epidemiológica. Dada la tendencia observada en los últimos años. a reducir la duración de las estancias y potenciar programas de alta precoz. con el fin de incrententar la eficiencia. deberian estimarse densidades de incidencia e incluir las IN diagnosticadas tras el alta para realizar comparaciones válidas entre diferentes centros y períodos de ticmpo.

Palabras clave: Infección Nosocomial. Medidas de frecuencia. Tasas de infección. Criterios diagnósticos. Localización de la infección. Comparabilidad.

Correspondencia

María José Rabanaque.

Departamento de Microbiología, Medicina Preventiva y Salud Pública.

Facultad de Medicina. Universidad de Zaragoza.

C/ Domingo Miral s/n.

50009 ZARAGOZA

FAX: (976) 761762

\section{ABSTRACT \\ Nosocomial Infection in Surgical Patients. Problems when Measuring and Comparing Results}

Background: What is striking when studying the frequency of nosocomial infection $(\mathrm{NI})$ is the variability of the study data. Different frequency indicators and infection criteria are used for estimates and these make it difficult to compare works. The aim of this work is to estimate the frequency of hospital infection by using different indicators to compare the results.

Methods: A market study was carried out including patients admitted to four surgical units over the period of one year. The following indicators were used: proportion of patients infected, cumulative number of cases of infection and density of number of cases. The infections were detected through active search and included those acquired in Intensive Care Units and those diagnosed after patients had been discharged from hospital.

Results: A total of $14.5 \%$ of the patients suffered NI and $5 \%$ of the infections were diagnosed after discharge from hospital. In $38.5 \%$ of the cases of infection a microbiological study was not requested. The General Surgery Unit had the highest figures for the three indicators. Nevertheless, the magnitude of the differences between services was modified in line with the indicator used.

Conclusions: The real percentage of patients with $\mathrm{Nl}$ is higher than the values given by the usual monitoring systems. Given the trend witnessed over recent years whereby the length of hospital stays is being reduced and early discharge progranmes promoted with the aim of increasing efficiency, densities for the number of cases should be estimated and these should include the NI cases diagnosed after hospital discharge in order to make valid comparisons between different institutions and periods of time.

Key words: Nosocomial infection. Frequency measurements. Infection rates. Diagnosis criteria. Localisation of the infection. Comparability. 


\section{INTRODUCCIÓN}

Al estudiar la frecuencia de infección nosocomial (IN), llama la atención la variabilidad de los datos publicados. La Encuesta Nacional de Prevalencia, realizada en 1986 , señalaba que el $11,2 \%$ de los pacientes ingresados en nuestros hospitales padecían algún proceso de infección hospitalaria'. Según los datos del proyecto EPINE², en 1994, la prevalencia de pacientes con IN se situaba en el 7,24\%. El Ministerio de Sanidad y Consumo informa que entre un 3 y un $14 \%$ de los pacientes ingresados en hospitales de agudos adquieren una $\mathrm{IN}^{3}$.

Su frecuencia es difícil de estimar, varía de unos hospitales a otros ${ }^{2,4}$ y dentro del mismo hospital entre los diferentes servicios, siendo mayor en los de cirugía, cuidados intensivos, prematuros o quemados 5 . Estas diferencias pueden ser debidas a variaciones reales en frecuencia de IN entre centros, relacionadas con las características del hospital y con las de los pacientes ingresados en el mismo, tales como edad, patologías atendidas, frecuencia de enfermedades subyacentes y factores de riesgo, tipología de procedimientos clínicos o intensidad diagnóstica y terapéutica aplicada. Así mismo deben considerarse otros factores relacionados con la metodología utilizada para estimar la frecuencia en los diferentes estudios ya que pueden justificar, al menos parcialmente, las diferencias entre resulta$\operatorname{dos}^{6,7}$.

Entre las diferencias metodológicas, se encuentran la definición de los indicadores utilizados para estimar la frecuencia del problema; los criterios diagnósticos de infección; el diseño aplicado en los estudios prospectivos, retrospectivos o transversales, y los métodos de vigilancia aplicados en la detección de casos. No puede olvidarse que las tasas varían sustancialmente en función del método de vigilancia utilizado, siendo conocido que en los hospitales en los que se ponen en marcha programas activos de vigilancia se produce un aparente incremento de tasas debido a un aumento en la detección de las infecciones existentes $\mathrm{s}^{6,8-10}$.

Por otra parte, en el caso de los pacientes quirúrgicos que son atendidos tras la intervención en unidades de cuidados intensivos (UCls), se consideran por una parte las infecciones diagnosticadas en los servicios quirúrgicos y por otra las presentadas en las propias UCIs, por lo que los mismos pacientes forman parte de los denominadores de las tasas de infección calculadas para ambos servicios.

Otro hecho a destacar es que en la estimación de la frecuencia de IN no suelen ser consideradas las infecciones adquiridas en el hospital y diagnosticadas tras el alta de los pacientes, por lo que se suele infravalorar el problema. Este hecho es más importante a medida que se tiende a reducir la duración de la estancia hospitalaria y muchas IN, fundamentalmente las quirúrgicas, se manifiestan cuando el paciente ha sido dado de alta.

Algunos de los fenómenos descritos pueden tener mayor relevancia en servicios quirúrgicos por presentar habitualmente cifras importantes de $\mathrm{IN}^{2}$; así por ejemplo, según datos del proyecto EPINE², en 1994, Cirugía General presentó una prevalencia de infecciones del 11,1\%, Neurocirugía del 13,96\% y Traumatología del $9,35 \%$. Por otra parte, los pacientes quirúrgicos son atendidos con cierta frecuencia en las UCIs donde adquieren infecciones y además pucden presentar un número importante de infecciones de herida quirúrgica cuya clínica se manifiesta tras el alta ${ }^{11}$

El trabajo que se presenta es un estudio prospectivo en el que se plantearon los siguientes objetivos: estimar la frecuencia de infección nosocomial en pacientes quirúrgicos y utilizar diferentes indicadores de infección, con el fín de comparar los resultados obtenidos con los mismos.

\section{MATERIAL Y MÉTODOS}

El trabajo se llevó a cabo en el Hospital Clínico Universitario de Zaragoza, hospital 
docente de 890 camas que sirve de centro de referencia para la atención especializada del área de salud n. ${ }^{\circ} 3$ de la Comunidad Autonóma de Aragón. Fueron incluidos todos los pacientes ingresados en los servicios de traumatología, cirugía general, cirugía cardiovascular y neurocirugía, desde el día uno de mayo de 1988 al 30 de abril de 1989.

Se realizó un estudio observacional prospectivo en el que se llevó a cabo el seguimiento de una cohorte de ingresos hospitalarios durante un año. Se estudió la incidencia de infección nosocomial en pacientes quirúrgicos, la distribución de las infecciones por localizaciones y el tipo de diagnóstico utilizado en las mismas. Igualmente se analizaron algunas variables que pueden estar relacionadas con la aparición de infección nosocomial como edad, enfermedades subyacentes y características de las intervenciones quirúrgicas. Como enfermedades subyacentes se consideraron los siguientcs procesos: cánccr, diabetes, insuficiencia renal, inmunodeficiencia, malnutrición, coma, infección comunitaria previa, infección hospitalaria previa y obesidad. Estos datos se recopilaron de la historia clínica, por lo que podrían infravalorar la presencia de comorbilidad.

Para cada ingreso se cumplimentó un protocolo en el que se recopilaron datos de identificación, administrativos, clínico-quirúrgicos y relacionados con la infección hospitalaria. Como datos clínico-quirúrgicos se consideraron, entre otros: diagnóstico principal al alta, patología subyacente, grado de contaminación de la intervención quirúrgica, cirugía programada o urgente, anestesia general o no general, duración de la intervención, reintervenciones, utilización de quimioprofilaxis, número de IN presentadas y fallecimiento o no del enfermo. En cuanto a los aspectos relacionados con la infección hospitalaria se recogieron los siguientes datos: localización de las infecciones, fecha y tipo de diagnóstico, muestra microbiológica diagnóstica, gérmenes causantes de la infección, factores de riesgo por localizaciones y tratamiento antibiótico previo y posterior a la aparición de infección.

La recogida de información se realizó en dos fases, una durante la estancia hospitalaria de los pacientes y otra tras el alta de los mismos, y fue realizada por una sola persona formada para tal fin.

Los casos de infección nosocomial cuya clínica se manifestó durante el ingreso de los pacientes, se detectaron utilizando un méto do de búsqueda activa ${ }^{12}$. Se llevó a cabo un seguimiento directo, en los servicios en estudio, desde el ingreso hasta el alta, recopilando información del personal de enfermería, consultando con el personal médico cuando se consideró necesario y revisando los partes de seguimiento diario. Simultáneamente se controlaron los informes microbiológicos de los servicios estudiados. Tras el alta hospitalaria, pasados al menos treinta días de la misma, se revisaron las historias clínicas de todos los pacientes con el fin de completar la información obtenida durante su estancia en el hospital, así como detectar los casos de IN cuya clínica se manifestó tras ser dados de alta.

Para estimar la frecuencia de infección se calcularon los siguientes indicadores $6,13,14$

a) Proporción de pacientes infectados $=$ (N. ${ }^{\circ}$ de pacientes infectados durante el período en estudio/ $\mathrm{N}{ }^{\circ}$ de ingresos durante el mismo período) $\times 100$; b) Incidencia acumulada de infección (IA) $=\left(\mathrm{N}^{\circ}{ }^{\circ}\right.$ de infecciones durante el período en estudio/N. ${ }^{\circ}$ de ingresos durante el mismo período) $\times 100$; c) Tasa de incidencia o Densidad de incidencia $(\mathrm{DI})=\left[\mathrm{n} .^{\circ}\right.$ de infecciones durante el período en estudio/ $\Sigma\left(N .^{\circ}\right.$ de estancias en el servicio + estancias en (UCI) $] \times 100$. I a incidencia acumulada de infección en este contexto, no es una proporción sino una razón, puesto que en el numerador se incluyen infecciones y no pacientes.

Para las diferentes medidas de frecuencia se calcularon intervalos de confianza fijando un nivel de confianza del $95 \%{ }^{15}$. 
Se estimaron razones de incidencia de infección entre servicios ${ }^{16}$, utilizando los indicadores de porcentaje de infectados y de densidad de incidencia. Como expuestos se consideraron los servicios con mayor frecuencia de infección.

En los numeradores se incluyeron las infecciones adquiridas por los pacientes durante su estancia en los servicios quirúrgicos estudiados y en las Unidades de Cuidados Intensivos. También se consideraron aquellas infecciones nosocomiales diagnosticadas tras el alta de los pacientes y que quedaron reflejadas en algún documento de la historia clínica, tales como informes de reingresos, informes de urgencias o datos de los seguimientos realizados en consultas externas. Es conocido que de las infecciones nosocomiales cuya clínica se manifiesta tras el alta no siempre queda constancia en documentos de la historia clínica, bien porque no son atendidas en los servicios hospitalarios o porque éstas no se registran, por lo que sabemos que las infecciones detectadas en nuestro estudio infravaloran la magnitud del problema.

Para el diagnóstico de infección nosocomial se aplicaron los criterios de los Centers for Disease Control de Atlanta ${ }^{17-19}$.

Fueron excluidos los enfermos con estancia en los servicios estudiados inferior a 48 horas, los que no pertenecían al área de hospitalización de los servicios descritos $\mathrm{y}$ aquellos de los que no se dispuso de información suficiente para cumplimentar el protocolo del estudio. Se excluyeron los pacientes con estancias inferiores a 48 horas, puesto que es muy difícil determinar si el origen de una infección que se manifiesta en las primeras horas de ingreso tiene origen comunitario u hospitalario ${ }^{18}$.

En cuanto a los denominadores, se consideraron como ingresos tanto los procedentes de fuera del hospital como los trasladados de otros servicios del centro. El concepto de ingreso utilizado no coincide exactamente con el administrativo y es necesario realizar algunas puntualizaciones.
Los reingresos por causa de infección hospitalaria y las readmisiones tras pases de fin de semana, no se consideraron nuevos ingresos, sino que se contabilizó el primer ingreso y se sumaron a éste todas las estancias hospitalarias ocasionadas. Igualmente cuando los enfermos fueron trasladados a las UCIs no se les consideró dados de alta y, de igual forma, su retorno al servicio no se incluyó como un nuevo ingreso, sumando las estancias en la UCI a las del servicio.

Se aplicaron los criterios comentados, junto con la inclusión de las IN adquiridas por los pacientes quirúrgicos en la UCI, y de las estancias en la misma, porque el objeto del estudio era estimar el porcentaje real de pacientes quirúrgicos que adquieren una IN, más que obtener datos de infección por servicio quirúrgico y UCI, indicadores estimados mensualmente por el servicio de medicina preventiva.

El tratamiento estadístico se realizó con el programa EPIINFO v5. Los tests aplicados fueron en la comparación de porcentajes $\chi^{2}$ y en la comparación de medias análisis de la varianza y las pruebas no parámetricas $U$ de Mann-Whitney y Kruskal-Wallis. Se aplicaron los pruebas no parámetricas cuando las condiciones de aplicación de las parámetricas no se cumplieron, fenómeno que se observó en numerosas ocasiones.

\section{RESULTADOS}

\section{Frecuencia de infección nosocomial}

Fueron registrados 4.044 ingresos de los que se excluyeron 386: 116 por permanecer en el servicio un tiempo inferior a 48 horas; 165 por pertenecer a otros servicios, 96 por no estar disponible la historia clínica y 9 por ser insuficiente la información disponible. Finalmente, el número de ingresos incluidos en el estudio fue de 3.658 , cuya distribución por servicios se presenta en la tabla 1 .

El $14,5 \%$ (530) de los pacientes ingresados padecieron alguna infección nosocomial. El número total de infecciones fue de 
los nacidos desde el 01.01 al 01.09 de 1994 . El primer caso del brote apareció el 15.12.1993.

c) el estudio incluye a vacunados y no vacunados.

d) la tasa de ataque de la cohorte fue superior al $5 \%$.

e) el n..$^{\circ}$ de niños de la cohorte fue elevado: 592 niños estudiados.

f) los sujetos vacunados por primera vez durante la epidemia fueron considerados según el status vacunal que tenían antes de ocurrir el brote, es decir como no vacunados.

g) asumimos que tanto la vacunación como las posibilidades de contagio durante el brote se produjeron de forma aleatoria entre el colectivo infantil.

Se consideró caso de sarampión a los niños que presentaron: erupción cutánea de 3 o más días de duración, fiebre igual o superior a los $380^{\circ} \mathrm{C}$ y al menos uno de los tres signos siguientes: tos, coriza o conjuntivitis ${ }^{6}$.

El sistema de información epidemiológica del distrito (censo de nacidos a partir del Registro de Metabolopatías y el Registro de Vacunaciones de Andalucía) permitió conocer el estado vacunal previo al brote del colectivo infantil, y la notificación de enfermedades de declaración obligatoria los casos presentados. Estos fueron notificados por el único pediatra existente en el municipio; a los niños sobre los que se carecía de información sobre su estado vacunal se les realizó una visita domiciliaria por parte de la trabajadora social con objeto de detectar vacunaciones administradas por otros centros o profesionales ajenos al municipio. Ninguno de ellos presentaba dicha eventualidad.

Se consiguió información del estado vacunal de la totalidad de los niños de la cohorte; solamente pudo existir un pequeño sesgo si algún niño vacunado enfermó y no fue atendido por el pediatra de la localidad, hecho que pensamos poco probable.

La eficacia vacunal (EV) es la capacidad que tiene una vacuna de inmunizar a una persona susceptible tras su administración, sin cmbargo cuando esta eficacia es determinada sobre el terreno equivale a la efectividad directa (ED) o efectos directos de la vacuna, y expresa el nivel de protección que ésta es capaz de conferir a la persona vacunada. Se calcula mediante la formula siguiente ${ }^{1,4,10}$ :

$$
\begin{aligned}
\mathrm{EV}=\mathrm{ED} & =(\mathrm{TAnv}-\mathrm{TAv}) / \mathrm{TAnv}= \\
& =1-\mathrm{TAv} / \mathrm{TAnv}
\end{aligned}
$$

donde TAnv es la tasa de ataque en no vacunados y TAv la tasa de ataque en vacunados.

A partir de TAnv y de TAv se determinaron igualmente:

- Los efectos indirectos (EI), relacionados con la disminución de la capacidad de transmisión del virus debido a la presencia de población vacunada. Es un efecto protector que la vacunación ejerce sobre los no vacunados denominado inmunidad de grupo o de rebaño ${ }^{2-4}$. Se calcula mediante la formula:

$$
\mathrm{EI}=1-\mathrm{TAnv} / \mathrm{TAc}
$$

Donde TAc es la tasa de ataque que la enfermedad produciría en una hipotética población control de similares características a la estudiada y en la cual no existiera ninguna persona vacunada. Se asume que el valor de la TAc es de 1 para el sarampión, ya que esta enfermedad afectaría al $100 \%$ de los susceptibles de la población infantil ${ }^{4}$.

- Los efectos totales (ET) expresan la reducción de la probabilidad de enfermar que presentan los vacunados debido a la confluencia del efecto directo de la vacuna en sí más el efecto indirecto o inmunidad de gru$\mathrm{po}^{2-4}$. Se calcula: 


$$
\mathrm{ET}=1-\mathrm{TAv} / \mathrm{TAc}
$$

- Los efectos medios (EM) son los que el programa de vacunación ejerce sobre el conjunto de la población estudiada ${ }^{2-4}$. Se mide mediante la formula:

$$
\mathrm{EM}=1-\mathrm{TAt} / \mathrm{TAc}
$$

donde TAt es la tasa de ataque en el total de los estudiados, pudiendo ser calculada mediante la formula

$$
\text { TAt }=(1-f)(\text { TAnv })+f(T A v)
$$

en la cual «f» es la fracción de población vacunada ${ }^{4}$.

\section{RESULTADOS}

Este municipio presenta una cobertura vacunal de triple vírica en la cohorte estudiada del $66^{\prime} 4 \%$ y la tasa de ataque global durante el hrote analizado fue del $8^{\prime} 3 \%$.(tabla 1 ).

La tasa de ataque para los vacunados fue del 1' $8 \%$ y del 21 ' $1 \%$ para los no vacunados. (tablas 2 y 3 ).

Tabla 1

Relación de niños censados, vacunados y enfermos y tasas de ataque del sarampión según año de nacimiento. Aznalcollar-1994

\begin{tabular}{|lcccc|}
\hline Año & Censados & Vacunados & Enfermos & Tasa ataque \\
\hline $1986(1)$ & 62 & $39(63 \%)$ & 4 & $6,5 \%$ \\
1987 & 100 & $56(56 \%)$ & 9 & $9 \%$ \\
1988 & 81 & $40(49 \%)$ & 12 & $14,8 \%$ \\
1989 & 73 & $51(70 \%)$ & 6 & $8,2 \%$ \\
1990 & 80 & $66(82,5 \%)$ & 4 & $5 \%$ \\
1991 & 83 & $67(81 \%)$ & 6 & $7,2 \%$ \\
1992 & 65 & $51(78 \%)$ & 1 & $1,5 \%$ \\
$1993(2)$ & 48 & $23(48 \%)$ & 7 & $14,6 \%$ \\
\hline Total & 592 & $393(66,4 \%)$ & 49 & $8,3 \%$ \\
\hline
\end{tabular}

(1) Nacidos a partir del 30.06 .1986

(2) Nacidos hasta el 01.09.1993.

Fuente: Registros de Metabolopatías, del Programa de Vacunación de Andalucía y del Sistema de notificación de Enfermedades de declaración obligatoria.

Tabla 2

Estado clínico y estado vacunal de la cohorte estudiada

\begin{tabular}{|lccc|}
\hline \multicolumn{1}{|c}{ Estado Vacunal } & Enfermos & Sanos & Total \\
\hline Vacunados & 7 & 386 & 393 \\
No vacunados & 42 & 157 & 199 \\
\hline Total & 49 & 543 & 592 \\
\hline
\end{tabular}

Tabla 3

Tasas de ataque calculadas en el brote epidémico

$\begin{array}{lr}\text { Cobertura vacunal o fracción de vacunados (f) } & 66,4 \% \\ \text { Tasa de ataque en la totalidad de la cohorte (TAt) } & 8.3 \% \\ \text { Tasa de ataque en vacunados (TAv) } & 1,8 \% \\ \text { Tasa de ataque en no vacunados (TAnv) } & 21,1 \% \\ \text { Tasa de ataque en población control (TAc) } & 100 \%\end{array}$


Tabla 1

Pacientes e infección nosocomial por servicios

\begin{tabular}{|lccccc|}
\hline Variables/Servicies & Tramatología & Cirugía General & Cardiovascular & Neurocinagia & GLOBAL \\
\hline N.o ingresos & 747 & 1768 & 667 & 476 & 3658 \\
N.o pacientes infectados & 92 & 306 & 72 & 60 & 530 \\
N.o infecciones & 109 & 374 & 86 & 88 & 657 \\
\hline
\end{tabular}

Incluidas las infecciones adquiridas en los servicios yuirúrgicos, en las unidades de cuidados intensivos y las diagnosticadas tras el alta hospitalaria y registradas en la historia clínica.

657, presentando infección múltiple el 19\% de los infectados. La media de infecciones por paciente infectado resultó ser de 1,2 y en los pacientes con infección múltiple de 2,2. El $14,4 \%$ (527) de los ingresados fueron atendidos en algún momento en la UCI.

Por servicios (tabla 2), los pacientes de cirugía general presentaron las cifras más ele- vadas de $\mathrm{IN}$, en los tres indicadores utilizados. El cociente del porcentaje de infectados entre este servicio y cardiovascular, que presentó los valores más bajos, fue de 1,60 (IC 95\% 1,26$2,04)$ y en el caso de la densidad de incidencia de 1,75 (IC 95\% 1,35-2,26) (tabla 3). Las diferencias entre los otros servicios prácticamente no se modificaron cuando se estimaron densidades de incidencia.

Tabla 2

Frecuencia de infección nosocomial por servicios según diferentes indicadores

\begin{tabular}{|lccc|}
\hline Senvicio & $\begin{array}{c}\text { *Pac. infectados } \\
\text { \% IIC) }\end{array}$ & $\begin{array}{c}{ }^{0} \text { I. Acumulada } \\
\%(I C)\end{array}$ & $\begin{array}{c}\text { \#Densidad de } I . \\
\text { \% (IC) }\end{array}$ \\
\hline Traumatología & $12,3(10,0-14,6)$ & $14,6(12,1-17,1)$ & $0.91(0,74-1,08)$ \\
Cirugía General & $17,3(15.5-19,1)$ & $21,1(19,2-23,0)$ & $1,49(1,34-1,64)$ \\
Cardiovascular & $10,8(8.5-13,1)$ & $12,9(10,4-15,4)$ & $0,85(0,67-1,03)$ \\
Neurocirugía & $12,6(9.6-15,6)$ & $18,5(15,0-22,0)$ & $0,97(0,77-1,17)$ \\
Global & $14.5(13,4-15,7)$ & $18,0(16,8-19,2)$ & $1,17(1,08-1,26)$ \\
\hline
\end{tabular}

Incluidas las infecciones adquiridas en los servicios quirúrgicos, en las unidades de cuidados intensivos y las diagnosticadas tras el alta hospitalaria y registradas en la historia clínica.

$*$ Porcentaje de pacientes infectados $=\left(N{ }^{\circ}\right.$ pacientes infectados $/ N{ }^{\circ}$ ingresos $) \times 100$.

"Incidencia Acumulada $=\left(\mathrm{N},{ }^{\circ}\right.$ de infecciones $/ \mathrm{N}{ }^{\circ}$ de ingresos $) \times 100$.

\# Densidad de Incidencia $=\left[\mathrm{N} .^{\circ}\right.$ de infeceiones $/\left(\mathrm{N} .^{\circ} \mathrm{dc}\right.$ estancias en el servicio + estancias en UCL) $] \times 100$.

Tabla 3

Razones de frecuencia de infección nosocomial entre servicios en función del indicador utilizado

\begin{tabular}{|lcc|}
\hline *Expuestos/"No expuestos & $\begin{array}{c}\text { \% pacientes infectados } \\
R R(I C \text { 95\%) }\end{array}$ & $\begin{array}{c}\text { Densidad de Incidencia } \\
\text { RR (IC 95\%) }\end{array}$ \\
\hline Cirugía General/Traumatología & $1,41(1,13-1,75)$ & $1,64(1,29-2,07)$ \\
Cirugía General/Cardiovascular & $1,60(1,26-2,04)$ & $1,75(1,35-2,26)$ \\
Cirugía General/Neurocingía & $1,37(1,06-1,78)$ & $1,54(1,16-2,03)$ \\
Neurocirugía/Traumatología & $1,02(0,75-1,39)$ & $1,06(0,76-1,47)$ \\
Neurocirugía/Cardiovascular & $1,17(0,85-1,61)$ & $1,14(0,81-1,60)$ \\
Traumatología/Cardiovascular & $1,14(0,85-1,53)$ & $1,07(0,78-1,45)$ \\
\hline
\end{tabular}

* Servicio con mayor incidencia." Servicio con menor incidencia. IC = Intervalo de Confianza 95\%. RR =Ie/Io. 
El cinco por ciento de las infecciones descritas fueron diagnosticadas tras el alta de los pacientes. Estas infecciones representaron un $10,1 \%$ del total de infecciones en traumatología; un $4,3 \%$ en cirugía general; un $2,3 \%$ en cardiovascular y un $4,5 \%$ en neurocirugía.

\section{Localización de la infección}

Las infecciones más frecuentes fueron las quirúrgicas, seguidas por las localizadas en vías urinarias, fenómeno que se observa en todos los servicios excepto en neurocirugía, donde las infecciones más frecuentes fueron las de vías bajas respiratorias y las urinarias (tabla 4). En el grupo de «otras» se incluyeron 10 infecciones de herida no quirúrgica, 7 flebitis, 4 peritonitis no operatorias, 4 hepatitis postranfusionales, 2 candidiasis orales, 2 faringo-amigdalitis y una infección vaginal.
En cuanto a la frecuencia de infección quirúrgica, infección superficial de la herida e infecciones quirúrgicas profundas, se diagnosticó infección en el 9,4\% de las intervenciones. Por grado de contaminación se observaron las siguientes incidencias acumuladas: cirugía limpia $5,5 \%$; limpia-contaminada $8,0 \%$; contaminada $19,6 \%$; sucia $22,2 \%$.

Las infecciones diagnosticadas tras el alta hospitalaria supusieron un $4,4 \%$ de las infecciones de herida quirúrgica; un $12,3 \%$ de las quirúrgicas profundas; un $2 \%$ de las urinarias; un $5,7 \%$ de las de vías respiratorias bajas y un $10 \%$ de otras localizaciones.

\section{Diagnóstico etiológico}

En este apartado hay que destacar que sólo en el 50 por ciento de las infecciones se dispuso de diagnóstico etiológico (tabla 5). En los resultados para cada una de las loca-

Tabla 4

Localización de las infecciones nosocomiales por servicios

\begin{tabular}{|c|c|c|c|c|c|c|c|c|c|c|}
\hline \multirow{2}{*}{ Localización } & \multicolumn{2}{|c|}{ Traumatología } & \multicolumn{2}{|c|}{ C. General } & \multicolumn{2}{|c|}{ Cardiovascular } & \multicolumn{2}{|c|}{ Neurocirugía } & \multicolumn{2}{|c|}{ GLOBAL } \\
\hline & $n$ & $(\%)$ & $n$ & $(\%)$ & $n$ & $(\%)$ & $n$ & $(\%)$ & $n$ & $(\%)$ \\
\hline Quirúrgica superficial & 50 & $(45,9)$ & 193 & $(51,6)$ & 24 & $(27,9)$ & 3 & $(3,4)$ & 270 & $(41,1)$ \\
\hline Quirúrgica profunda & 5 & $(4,6)$ & 43 & $(11,5)$ & 5 & $(5,8)$ & 4 & $(4.5)$ & 57 & $(8,7)$ \\
\hline Vías urinarias & 31 & $(28,4)$ & 65 & $(17,4)$ & 24 & $(27,9)$ & 32 & $(36,4)$ & 152 & $(23,1)$ \\
\hline Vías Respiratorias Bajas & 14 & $(12,8)$ & 54 & $(14,4)$ & 21 & $(24,4)$ & 33 & $(37,5)$ & 122 & $(18,6)$ \\
\hline Scpsis & 3 & $(2,8)$ & 11 & $(3,0)$ & 4 & $(4,7)$ & 8 & $(9,1)$ & 26 & $(4,0)$ \\
\hline Otras & 6 & $(5,5)$ & 8 & $(2,1)$ & 8 & $(9,3)$ & 8 & $(9,1)$ & 30 & $(4,5)$ \\
\hline TOTAL & 109 & $(100,0)$ & 374 & $(100,0)$ & 86 & $(100,0)$ & 88 & $(100,0)$ & 657 & $(100,0)$ \\
\hline
\end{tabular}

Incluidas las infecciones adquiridas en los servicios quirúrgicos, en las unidades de cuidados intensivos y las diagnosticadas tras el alta hospitalaria y registradas en la historia clínica.

Tabla 5

Estudio microbiológico por localizaciones

\begin{tabular}{|lccccccc|}
\hline \multicolumn{1}{|c}{ Diagnóstico } & IHQ \% & IQP \% & Urinarias \% & IVBR \% & Sepsis \% & Otras \% & GLOBAL \% \\
\hline No cultivo * & 44,5 & 26,3 & 32,9 & 58,2 & 11,5 & 43,3 & $38,5 \%$ \\
Resultado microbiológico (+) & 44,4 & 64.9 & 53,9 & 38,5 & 84,7 & 56,7 & $50,0 \%$ \\
Resultado microbiológico (-) & 11,1 & 8,8 & 13,2 & 3,3 & 3,8 & - & $11,5 \%$ \\
\hline
\end{tabular}

$\mathrm{IHQ}=$ Infección superficial de heriđá quirúrgica. IQP = Infección quirúrgica profunda. IVBR = Infección de Vías Bajas Respiratorias. * No realizado cultivo.

"Diagnóstico clínico y estudio microbiológico positivo.

\# Diagnóstico clínico y estudio microbiológico negativo. 
lizaciones llama la atención el importante número de infecciones superficiales de herida quirúrgica, $44,5 \%$, en las que no se realizó cultivo microbiológico.

\section{Algunas características de los pacientes y las intervenciones}

Al analizar la distribución por servicios de algunas variables que pueden estar relacionadas con la IN (tabla 6) destaca que la edad media de los pacientes atendidos fue de 53,5 $\pm 19,7$ años. La media de edad de los pacientes de neurocirugía fue inferior a la de los otros tres servicios y la de cardiovascular superior a la del resto $(\mathrm{p}<0,05)$.

En cuanto a las estancias medias, neurocirugía presentó medias significativamente más elevadas en estancia en el servicio y en UCI que los otros servicios $(p<0,05)$.

Por otra parte, el 4,9\% de pacientes fallecieron y el $29,7 \%$ presentaron patología subyacente. Traumatología, con un $2,9 \%$ de fallecidos, presentó los valores más bajos en esta variable $(p<0,05)$ y cirugía general y cardiovascular los más altos en porcentaje de pacientes con patología subyacente $(\mathrm{p}<0,05)$.

Se observaron grandes diferencias entre servicios en las variables relacionadas con las intervenciones quirúrgicas, siendo el servicio de cirugía general el que presentó el mayor porcentaje de intervenciones con anestesia general y el menor de intervenciones limpias $(\mathrm{p}<0,05)$.

\section{DISCUSIÓN}

El porcentaje de pacientes excluidos en nuestro estudio no fue importante, $9,5 \%$, y por tanto consideramos que no cuestiona la validez del mismo.

La frecuencia de infección nosocomial observada, $14,5 \%$ de los pacientes adquirie-

Tabla 6

Distribución de algunas variables por servicios

\begin{tabular}{|lccccc|}
\hline Variables & Traumatología & C. General & Cardiovascular & Neurocirugía & GLOBAL \\
\hline \% varones & 54,8 & 53,3 & 74,1 & 66,8 & 59,1 \\
$\mu$ edad \pm DE & $51,5 \pm 22,9$ & $52,3 \pm 19,3$ & $64,9 \pm 13,6$ & $44.9 \pm 16,2$ & $53.5 \pm 19.7$ \\
Estancia $\mu$ servicio $\pm \mathrm{DE}$ & $15,8 \pm 11,8$ & $13,7 \pm 13,2$ & $14,4 \pm 15,2$ & $17,7 \pm 18,2$ & $14.8 \pm 14,1$ \\
Estancia $\mu$ UCI \pm DE & $0,2 \pm 2,1$ & $0,5 \pm 2,0$ & $0,8 \pm 2,1$ & $1.5 \pm 5,1$ & $0.6 \pm 2,6$ \\
\% defunciones * & 2.9 & 5,3 & 5,8 & 4,0 & 4,9 \\
\% pac. patología subyacente & 14,2 & 33,9 & 44,7 & 17,0 & 29,7 \\
\% pacientes UCI \# & 3,5 & 12,4 & 27,9 & 19,5 & 14,4 \\
\% pacientes intervenidos & 81,8 & 89,8 & 74,4 & 46,2 & 79,7 \\
\% pac. varias intervenciones\$ & 5,1 & 4,6 & 12,1 & 8,2 & 6,2 \\
\% intervenciones urgentes & 32,9 & 30,4 & 10,7 & 12.7 & 26,2 \\
\% anestesia general & 73,5 & 97,4 & 64,7 & 90,5 & 73,8 \\
\% cirugía limpia & 92,0 & 31,7 & 90,3 & 94,5 & 59,0 \\
Duración $\mu$ intervención \pm & $91 \pm 56$ & $105 \pm 69 \mathrm{DE}^{00}$ & $129 \pm 85$ & $174 \pm 87$ & $111 \pm 74$ \\
\hline
\end{tabular}

$\mu=$ media.

* Porcentaje de pacientes que fallecieron.

"Porcentaje de pacientes con patología subyacente

\# Porcentaje de pacientes que estuvieron ingresados en la UCI.

\$ Purcentaje de pacientes con más de una intervención quirúrgica

${ }^{\text {"ki }}$ Duración media

$\pm D E$ de las intervenciones, en minutos. 
ron infección durante su estancia en el servicio quirúrgico o en la UCI, 18 infecciones por cada cien pacientes y 1,17 infecciones por cien estancias; esta cifra es superior a la de otros trabajos, destacando por su importancia el estudio NNIS ${ }^{20}$ con 3,5 infecciones por cada cien altas y el proyecto SENIC $^{13}$ con $5,7 \%$ infecciones por cada cien ingresos. En nuestro país diferentes estudios muestran datos de incidencia acumulada que va de 3,2 a 12,1 infecciones por cada cien ingresos ${ }^{5,10,21-24}$. El proyecto EPINE de 1994 describe, para el conjunto de los hospitales, una prevalencia de infectados de $7,24 \%$ y de 8,34 infecciones por cada cien pacientes $^{2}$. Por servicios (tabla 2) nuestras estimaciones fueron superiores a los datos de prevalencia de infecciones aportados por este mismo estudio: cirugía general $11,33 \%$; traumatología $6,76 \%$; neurocirugía $5,05 \%$; cirugía cardiaca $7,21 \%$ y cirugía vascular $10,36 \%$.

Por localizaciones las infecciones de herida quirúrgica fueron las más frecuentes. Este hecho ha sido descrito en otros estu$\operatorname{dios}^{25,26}$. Las tasas de infección quirúrgica, en general y en cirugía limpia, fueron superiores a los datos de 1994 del proyecto EPI NE. No podemos olvidar que en nuestro estudio, en el $44,5 \%$ de las infecciones de herida quirúrgica no se realizó cultivo, fenómeno que puede producirse en muchos centros hospitalarios. Igualmente, como se ha comentado, fueron incluidas las infecciones diagnosticadas tras el alta que fueron registradas en la Historia Clínica, fuente de información que sabemos insuficiente para estimar la importancia del fenómeno, pero que fue el sistema por el que se detectaron el $4,4 \%$ de las infecciones superficiales de herida y el $12,3 \%$ de las infecciones quirúrgicas profundas, valores nada despreciables. Este seguimiento es y será en un futuro, de gran interés, dada la implantación progresiva de programas de cirugía ambulatoria y de corta estancia.

I as infecciones urinarias tuvieron menos importancia que la descrita generalmente, hecho que puede ser debido a que en el estudio no se incluyeron los servicios de urología, ginecología ni obstetricia en los que estas infecciones son frecuentes.

La mayor incidencia de IN hallada en nuestro estudio en relación a la descrita en otros trabajos podría estar justificada por las siguientes razones:

a) La inclusión solamente de pacientes quirúrgicos que, como es conocido, tienen un riesgo elevado de padecer infección nosocomial ${ }^{3,5,8,9,24}$. Por otra parte, el hospital en el que se ha llevado a cabo el estudio es un centro universitario con un número importante de camas en el que se llevan a cabo procedimientos diagnósticos y terapéuticos de gran complejidad. En este tipo de hospitales suelen presentarse tasas más elevadas de infección ${ }^{3,4,27}$. Igualmente en la comparación de datos de infección habría que tener en cuenta las características de los pacientes incluidos en los estudios, tales como diagnósticos, edad y factores de riesgo ${ }^{8,27,28}$, siendo difícil asegurar la comparabilidad de los pacientes estudiados ${ }^{29}$. Los servicios incluidos presentaron entre sí diferencias considerables en algunas variables tales como edad media de los pacientes, frecuencia y tipo de patologías subyacentes presentadas por los mismos, porcentaje de intervenciones urgentes y duración de las intervenciones. Este hecho puede explicar, al menos parcialmente, las diferentes tasas de infección observadas. Algunas variables como edad, características de los pacientes y duración de la estancia hospitalaria (tabla 6) hacen cuestionarse si los pacientes atendidos en estos centros pueden seguir considerandose enfermos agudos.

b) Metodológicamente sc adoptaron una serie de criterios que afectaron a los numeradores y denominadores de las tasas. En este sentido no podemos olvidar que en las infecciones consideradas están incluidas las adquiridas en las UCIs, por lo que las tasas de infección descritas no son propiamente por servicio quirúrgico. Esta decisión se 
adoptó por varias razones: primero porque el objetivo del trabajo no era tanto obtener tasas por servicios como conocer realmente la magnitud del problema de la IN en pacientes quirúrgicos; $y$ en segundo lugar porque no suele resultar fácil en pacientes que sufren traslados entre servicios quirúrgicos y UCIs, diferenciar el servicio en el que se adquiere la infección.

Según las estadísticas habituales del servicio de Medicina Preventiva, en 1988 la incidencia acumulada de infección para el total del hospital fue de 7,18 infecciones por cada cien ingresos. Para los servicios en estudio en ese mismo año los datos de incidencia acumulada fueron: traumatología $9,9 \%$ (estudio: 14,6 ); cirugía general $15,3 \%$ (estudio: 21,1 ); cardiovascular 9,3\% (estudio: 12,9); neurocirugía 12,7\% (estudio: $18,5)$ y para el conjunto de los cuatro servicios 12,9 (estudio: 18,0). Estos valores son algo más elevados que los aportados por el proyecto EPINE en los últimos años ${ }^{2}$, diferencia justificada por el método de vigilancia y búsqueda activa de los casos de IN. No podemos olvidar además, las limitaciones en la comparabilidad dado que el proyecto EPINF es un estudio de prevalencia, recopila datos de hospitales de características diversas y los datos son posteriores al año de nuestro trabajo. En los servicios en estudio las tasas de infección también han descendido considerablemente en los últimos años.

En cuanto a los datos de densidad de incidencia, debe destacarse que existen muy pocos estudios en nuestro medio y que, como han descrito Delgado et $\mathrm{l}^{30}$, los datos de densidad de incidencia son los más pertinentes para realizar comparaciones entre servicios y hospitales. En cl trabajo presentado las diferencias entre servicios sufrieron algunas variaciones cuando se calcularon densidades de incidencia (tabla 3). Es conocido que, al estimar datos de porcentaje de infectados o de incidencia acumulada de infección, tienden a presentar valores más altos los servicios con estancias más prolon- gadas, puesto que sus denominadores serán menores y muy probablemente los pacientes atendidos más complicados y con mayor riesgo de infección. Como puede observarse en la tabla 6 los pacientes de cirugía general presentaron la media de estancia hospitalaria más baja, y los de neurocirugía la más alta.

El método utilizado en la detección de casos de infección, seguimiento activo y directo en los servicios, la inclusión de criterios clínicos en el diagnóstico y el considerar todas las localizaciones de infección, han dado lugar a considerar un número de infecciones mayor que si se hubiesen aplicado otros criterios $^{6,8-10}$. Como se ha descrito, es frecuente la falta de diagnóstico microbiológico en las IN, bien porque este no se realiza $(38,5 \%)$ o porque, aún existiendo clínica evidente, este resultado es negativo $(11,5 \%)$, resultados similares a los de otros estudios ${ }^{31}$. Estos hechos ponen de manifiesto que aquellos centros en los que se realice un mayor seguimiento de la IN serán los que presenten las cifras más elevadas.

Por otra parte y en comparación con otros estudios, los denominadores se han visto reducidos, debido a la exclusión de los ingresos cuya estancia en los servicios fue inferior a 48 horas y a que no han sido considerados como ingresos nuevos los reingresos tras fin de scmana, ni reingresos por infección nosocomial, ni los traslados de entrada de UCI, en el caso de pacientes ingresados previamente en el mismo servicio. Se aplicó este criterio porque consideramos que para calcular porcentaje de infectados e IA de infecciones era la población a riesgo real. Considerando en el denominador el número de ingresos administrativos, el porcentaje de pacientes infectados, a nivel global, para el mismo período de estudio pasaría del $14,5 \%$ al $12,3 \%$ y por servicios se reduciría del siguiente modo: traumatología pasaría del $12,3 \%$ al $11 \%$, cirugía general del $17,3 \%$ al $14,3 \%$, cirugía cardiovascular del $10,8 \%$ al $9,5 \%$ y neurocirugía del $12,6 \%$ al $9,3 \%$. 
Tampoco podemos olvidar que, como ha sido descrito, en el estudio se incluyeron tambien las IN cuya clínica se manifestó tras el alta hospitalaria y que fue posible detectar, bien porque constaban en los documentos de la historia clínica, correspondientes a seguimiento en consultas externas, bien porque los pacientes reingresaron por esa causa. Estas IN no suelen ser consideradas en la mayoría de los trabajos publicados. Con respecto a este tema se plantean algunas consideraciones:

El número de infecciones diagnosticadas tras el alta fue importante, representando el $5 \%$ del total, y los valores más clevados por servicios correspondieron a traumatología con un $10 \%$. Hay que tener en cuenta, sin embargo, que la detección por historia está sesgada por el grado de cumplimentación de las misma y que por tanto el número de infecciones diagnosticadas tras el alta en nuestro estudio habrá sido probablemente infravalorado. $\mathrm{Ha}$ sido descrita la importancia numérica de las infecciones hospitalarias diagnosticadas tras el alta del paciente, aceptándose que entre un 25 y un $60 \%$ de las infecciones de herida quirúrgica se manifiestan cuando el enfermo ha abandonado el hospital ${ }^{11,32-35}$.

Dado que se tiende a reducir la duración de la estancia hospitalaria parece necesario establecer algún sistema de control tras el alta de los pacientes, ya que será cada vez más frecuente que las infecciones adquiridas en los hospitales, y particularmente las quirúrgicas, se manifiesten tras el alta de los enfermos. De lo contrario, aparentes reducciones en la tasa de infección nosocomial podrían ser atribuidas a una insuficiente vigilancia.

De los resultados presentados y la discusión extraemos las siguientes conclusiones:

- El porcentaje real de pacientes quirúrgicos que sufren una IN cs superior a los datos habitualmente aportados para nuestro país, por lo que continúa siendo necesario desarrollar y potenciar aquellas medidas preventivas que sean más efectivas y eficientes en el control de la IN.
- La estimación de densidades de incidencia de infección permite realizar comparaciones cuando existen diferencias en duración de estancias. En el futuro debería hacerse un esfuerzo por estimar densidades de incidencia si se quieren comparar datos, no sólo de diferentes centros y servicios, sino también de los mismos servicios a lo largo del tiempo. Este aspecto es importante si se considera que las políticas de alta precoz, y de programas de corta estancia, iniciadas en los últimos años, limitan la comparabilidad de otros indicadores.

- El 5\% de las IN incluidas fueron diagnosticadas tras el alta y referenciadas en la historia clínica, por lo que el número real será más elevado. Resulta necesario realizar el control de la IN diagnosticadas tras el alta de los pacientes, sobre todo en infecciones quirúrgicas, si realmente se quiere conocer la magnitud y evolución del problema.

- No se pueden obviar las limitaciones existentes en la comparabilidad de los datos de IN entre hospitales y entre servicios, tanto por problemas metodológicos como por diversidad entre las características de los centros y de los pacientes atendidos. Este hecho es de gran importancia por la utilización que se hace de la IN como indicador de calidad de resultados. Parece necesario ser especialmente cautos a la hora de realizar comparaciones, evitando clasificaciones de hospitales y servicios en función de este indicador.

\section{AGRADECIMIENTOS}

Agradecemos la colaboración del personal de los servicios quirúrgicos en los que se realizó el estudio y del servicio de documentación y archivos.

\section{BIBLIOGRAFÍA}

1. Ministerio de Sanidad y Consumo. Encuesta Nacional de Prevalencia de la Infección Hospitalaria y de Consumo de Antimicrobianos. Madrid: Dirección General de Planificación Sanitaria; 1986. 
2. Grupo de trabajo EPINE. Prevalencia de las infecciones nosocomiales en los hospitales españoles. EPINE 1990-1994. Barcelona: Sociedad Española de Higiene y Medicina Preventiva Hospitalarias y grupo de trabajo EPINCAT; 1995.

3. Ministerio de Sanidad y Consumo. Informe sobre infección hospitalaria. Med Clín 1994: 102; 2024.

4. Allen JR, Hightower AW, Martin SM, and Dixon RE. Secular trends in nosocomial infections: 1970-1979. Am J Med 1981; 70: 389-392.

5. Grupo de Trabajo EPINCAT. Prevalencia de las infecciones nosocomiales en Cataluña. (I) Infecciones y factores de riesgo. Med Clín 1990; 95 : $41-52$

6. Freeman $\mathfrak{J}$ and McGowan JE. Methodologic issues in hospital epidemiology: I. Rates, CaseFinding and interpretation. Rev Infect Dis 1981; 3(4): 658-667.

7. Freeman $J$ and McGowan JE. Differential Risks of Nosocomial Infection. Am J Med 1981; 70: 915-918.

8. Thoburn R, Fekety FR, Cluff LE and Melvin VB. Infections Acquired by Hospitalized Patients. Arch Intern Med 1968; 121: 1-10.

9. Brawley R.L, Weber DJ, Samsa GP and Rutala WA. Multiple nosocomial infections an incidence study. Am J Epidemiol 1989; 130(4): 769-780.

10. Miralles R, Force LI, Verdaguer A, Torres JM, Serrano R, Pérez Vidal R, et al. Incidencia de la infección nosocomial. Comparación de dos sistemas de vigilancia: seguimiento clínico frente a seguimiento microbiológico. Med Clín (Barc) 1989: 92: 652-654.

11. Burns SJ, Dippe SE. Postoperative wound infections detected during hospitalization and after discharge in a community hospital. Am J Infect Control 1982: 10: 60-65.

12. Haley RW, Schaberg DR, Von Allmen SD and McGowan JE. Estimating the extra charges and prolongation of hospitalization due to nosocomial infections: a comparison of methods. J Infect Dis 1980; 14(2): 248-257.

13. Haley RW, Culver DH, White JW, Morgan WM and Emori TG. The Nationwide nosocomial infection rate. A new need for vital statistics. Am J Epidemiol 1985; 121(2): 159-167.

14. Bueno Cavanillas A, Delgado Rodríguez M, Cueto Espinar A y Gálvez Vargas R. Vigilancia epidemiológica de la infección hospitalaria. Rev Clín Esp 1987; 181(2): 92-97.
15. Daniel WW. Bioestadística. Base para $\mathrm{cl}$ análisis de las ciencias de la salud. 3? ed. Mexico: Editorial Limusa S.A.; 1990.

16. Ahlbom A. Biostatistics for epidemiologists. Boca Raton (Florida): Lewis Publishers; 1993. p. 6470 .

17. Center for Disease Control: Outline for surveillance and control nosocomial infections. CDC US Department of Health and Human Services. Georgia: Public Health Service; 1974.

18. Definiciones del CDC de infección nosocomial. Bol Microbiol Semanal 1988; 27. p. 1-5.

19. Definiciones del CDC de infección nosocomial. Bol Microbiol Semanal 1988; 30-31.

20. Dixon RE. Effect of infections on hospital care. Ann Intern Med 1978; 89(2): 749-753.

21. Carrasco Asenjo M, Delgado García A, Fernández Pérez C, Prieto Valiente L, Jimeno Maestro J y Andradas Aragonés E. Vigilancia epidemiológica de la infección hospitalaria. Análisis preliminar de una serie de cinco años. Med Clín 1990; 95: 201-206.

22. Ferrer P, Micheo C, Ramón JM, Peyra R, Tortras $\mathrm{N}$, Vidal T et al. Estudio prospectivo y metodología en el control informatizado de la infección hospitalaria. Enf Infecc Microbiol Clín 1987; 5(10): 595-602.

23. Carrasco M, Jimeno J, Aguado A, Magro R, Castillo O, Martínez F et al. El control de la infección hospitalaria en un hospital de 400 camas. Enf Infecc Microbiol Clín 1987; 5(10): 603-608.

24. Sáenz Gonzalez MC, Rodrigo Sánchez N, Gutierrez Fisac JL, Valero Juan L, Núñez Mateos JC y Meléndez Marugán D. Incidencia de la infección hospitalaria en un hospital universitario. Med Clín 1989; 92: 213-216.

25. Alonso Prieto MI, Castrodeza Sanz JJ. Coladas Rodríguez $\mathrm{N}$ y López Encinar P. Evaluación de la Infección Nosocomial en el hospital y su repercusión en los costes sanitarios. Rev Salud Pública Castilla y León 1994; 3: 29-37.

26. Maradona HidalgoA, Cartón Sánchez JA y Pérez del Molino G. Experiencia y valoración de los estudios de prevalencia de la infección nosocomial en un hospital de tercer nivel. Rev Clín Esp 1989; 184: 61-64

27. Brachman PS, Dan B, Haley RW, Hooten TM, Garner JS, Allen JR. Infecciones quirúrgicas nosocomiales, frecuencia y costo. Surg Clin North Am 1980; 1: 13-24.

28. Fabry J, Meynet R, Joron MT, Sepetjan M, Lambert DC and Guillet R. Cost of nosocomial infec- 
tions: analysis of 512 digestive surgery patients. World J Surg 1982; 6(3): 362-365.

29. Haley RW, Hooton TM, Culver DH, Stanley RC, Emori TG, Hardison CD et al. Nosocomial Infections in U.S. Hospitals, 1975-76. Estimated Frequency by Selected Characteristics of Patients. Am J Med 1981; 70: 947-957.

30. Delgado Rodríguez M, Sillero Arenas M, Rodríguez-Contreras Pelayo R y Gálvez Vargas R. Sobre la medición de la infección hospitalaria. Med Clín (Barc) 1990; 94: 271-274.

31. Grupo de Trabajo EPINCAT. Prevalencia de las infecciones nosocomiales en Cataluña. (II) Gérmenes y antimicrobianos. Med Clín 1990; 95: $161-168$
32. Brachman PS. Epidemiology of Nosocomial In fections. En: Bennett JV, Brachman PS. eds. Hospital Infections. 2.a ed. Boston/Toronto: Little, Brown and Company; 1986. p. 3-16.

33. Palmer MB. Manual de control de infecciones. Madrid: Emalsa SA, Interamericana; 1986.

34. Law DJW, Mishriki SF and Jeffery PJ. The importance of surveillance after discharge from hospital in the diagnosis of postoperative wound infection. Ann Royal College Surgeons England 1990; 72 : 207-209.

35. Brown RB, Bradley S, Opitz E, Cipriani D, Pieczarka R and Sands M. Surgical wound infections documented after hospital discharge. Am J Infect Control 1987; 15: 54-58. 